Hans Reithofer (2009): Verkörperte Feldforschung als Ethnologe und Missionar. In: Elfriede Hermann, Karin Klenke und Michael Dickhardt (Hg.): Form, Macht, Differenz. Motive und Felder ethnologischen Forschens. Göttingen: Universitätsverlag Göttingen, 357-370. Doi: 10.17875/ gup2020-1297

\title{
Verkörperte Feldforschung als Ethnologe und Missionar
}

\author{
Hans Reithofer
}

\section{Einleitung}

Missionare und Ethnologen sind für viele wie Feuer und Wasser, und eine Vermischung der beiden Rollen deshalb so ätzend wie Feuerwasser. In plakativen Stereotypen erscheinen die einen als Kulturzerstörer, die anderen als Kulturversteher und -bewahrer. Trotz dieser Klischees sehe ich für meinen Teil keine Notwendigkeit, meine Vergangenheit als Steyler Missionar (offiziell: Missionar der „Gesellschaft des Göttlichen Wortes“ bzw. „Societas Verbi Divini“, kurz SVD) zu verheimlichen - und keinen inhärenten Widerspruch zwischen beiden Rollen, die ich zumindest für die zweijährige Dauer meiner Feldforschung im westlichen Hochland von Papua-Neuguinea (PNG) ausgeübt habe. Andere haben das jahrzehntelang getan, ohne dabei schizophrene Züge zu entwickeln (siehe unten).

Es soll hier aber nicht die existentielle Vereinbarkeit beider Rollen demonstriert werden. Vielmehr möchte ich über die Besonderheiten ethnologischer Feldforschung reflektieren, wenn man gleichzeitig - wie in meinem Fall - Ethnologe und katholischer Missionar ist: Welche besonderen Herausforderungen und Probleme ergeben sich daraus für die Forschung in methodischer, ethischer und (macht-)politischer Hinsicht? Als Impulsgeber sollen dabei die Überlegungen einer überaus forschungsstarken Ethnologin dienen, die - wie sie selbst sagt - seit (mittlerweile mehr als) 30 Jahren aus- 
gesprochen gerne auf Feldforschung geht: Brigitta Hauser-Schäublin (2002:73). Am Schluss soll die häretische (?) Frage stehen, ob und inwieweit Missionare die besseren Ethnologen abgeben (können).

\section{Verkörperte Feldforschung}

Ihren Beitrag zur Neufassung der Feldforschungen (Fischer 2002) übertitelt HauserSchäublin mit: „Gender: Verkörperte Feldforschung", weil Feldforschung für sie immer Verkörperung bedeutet und damit den unausweichlichen Rückbezug auf das eigene Gender, den „Schnittpunkt von Hautfarbe, Kulturzugehörigkeit, Religion, Sprache und Sprechvermögen, zugeschriebenem Geschlecht, Sexualität, Alter und sozialem Status" (2002:85). Dieses Gender-Profil bestimme wesentlich die Rolle der Ethnologin oder des Ethnologen in der Gastgebergesellschaft und damit auch die Forschungssituation. Daraus ergebe sich die Notwendigkeit, subjektive Faktoren des Forschens offenzulegen, weil sie die Sichtweisen, Standpunkte und die Art der Wissensproduktion maßgeblich beeinflussten (2002:92, in Rückbezug auf Haraway). Sie selbst hat diese Forderung nicht nur vom Anfang ihrer Forscherlaufbahn an erhoben (1976:9), sondern auch umgesetzt (z. B. 1977:12-18), wie das gerade auch Ethnologinnen vor ihr (und lange vor der Writing Culture-Debatte) gemacht haben.

In meiner Feldforschung in der Südlichen Hochlandprovinz war mein Gender-Profil maßgeblich mitbestimmt durch die Tatsache, dass ich katholischer Priester war. Auf diesen Aspekt möchte ich mich hier auch konzentrieren. Ich mache in meiner Dissertation keinen Hehl daraus, dass bei meiner Ankunft die - ganz überwiegend katholische - Bevölkerung des gastgebenden Dorfes Ipisam eine beeindruckende Willkommensfeier abhielt für ihren neuen „Stationspfarrer“ und die Redner sich beglückwünschten, dass durch mich nun die kleine Buschstation in den Status einer Pfarre erhoben wurde (Reithofer 2006:13). Diese Hervorhebung meiner Rolle als Priester („Father John“) hielt sich durch bis zu meiner Abreise, wie etwa das Abschiedslied erkennen lässt, das die katholische Jugend im Jahr 2000 für mich getextet hat:

Bipo ol lain Ipisam

ol i bin bihainim lo istap,

tasol God i salim Father John,

yu bin kam long Ipisam.

Yu bin stretim laip bilong mi.

Nau yu laik go long ples bilong yu.

Mipela i tok gutbai.
Früher lebten die Leute von Ipisam

nach dem Gesetz [der Ahnen],

Gott aber sandte Father John,

und du kamst nach Ipisam.

Du hast mein Leben "gerade gerichtet".

Nun willst du nach Hause zurück.

Wir sagen "Auf Wiedersehen!” 
Die starke Fokussierung auf mein Priestersein war angesichts meines Forschungsinteresses von besonderer Brisanz, da ich die Prozesse der Rezeption des Christentums untersuchen wollte und damit auch das Verhältnis der vorchristlichen zur christlichen Religion. Sollte ich die Funktion eines Priesters ausüben - und ich war durchaus gewillt, dieser Erwartung meiner (katholischen) Gastgeber zu entsprechen - würde ich erheblichen direkten Einfluss auf meinen Forschungsgegenstand ausüben; zu dieser Problematik später.

Meine für jedermann präsente priesterliche Identität war ein zweischneidiges Schwert: Sie erleichterte mir den Zugang zu Mitgliedern der katholischen Gemeinde und legte von Anfang an den Grund für belastbare, vertrauensvolle Beziehungen, während sie zugleich den Zugang zu den stark anti-katholisch eingestellten SiebentenTags-Adventisten (STA) erheblich erschwerte. Das Timing meines Aufenthalts (19982000) verschärfte die Sache noch, weil die bevorstehende Jahrtausendwende auch in dem abgelegenen Hochlandtal Honda die millenaristischen Spekulationen beflügelte. Was Kristin Kocher Schmid (1999:23-24) über Millenniums-Diskurse und „symbolic anthropologists" schreibt, traf womöglich auf mich - als Ethnologen und Missionar - noch mehr zu: Ich selbst wurde für die Leute im Tal zu einem Symbol, einem Zeichen, dessen wahre Bedeutung sie immer wieder neu zu eruieren suchten. Was war der eigentliche Grund meiner Anwesenheit, und warum war ich gerade jetzt gekommen? Man erzählte mir etwa, dass man mich in adventistischen Kreisen für einen der falschen Propheten hielt, die gemäß biblischer Vorhersage (Matthäus 24:24) der Wiederkunft Christi vorausgingen, um die Gläubigen vom rechten Weg abzubringen. Manche Adventisten mieden mich deshalb wie der Teufel das Weihwasser. Als ich im Januar 2000 für einige Wochen die Forschung für einen Heimaturlaub unterbrach, vermuteten viele (wie ich später erfuhr), dass ich für die Wiederkunft Christi mit meiner eigenen Familie vereint sein wollte. Meine Anwesenheit und mein Status als Amtsträger der katholischen Kirche heizten also den lokalen Millenniums-Diskurs an, der ebenfalls zu meinen Untersuchungsgegenständen gehörte (vgl. Reithofer 2006:310-330), und schränkten die Gesprächsbereitschaft darüber ein.

Hier klingt schon eine grundsätzlichere Problematik durch: Mein Priesterstatus verschaffte mir eine Autorität, die mir nicht immer bewusst war. Welches Gewicht meinen Worten und Handlungen zugemessen und welche Bedeutung ihnen gegeben wurde, wurde mir oft erst viel später deutlich. Vielleicht habe ich diese Autorität auch deshalb unterschätzt, weil ich oft hörte, dass man mit mir ganz anders reden könne als mit den anderen Missionaren, die jeweils nur kurze Besuche machten.

Als Amtsträger einer christlichen Kirche war ich jedenfalls - und dies für Christen wie Nicht-Christen gleichermaßen - ein Repräsentant der "neuen Zeit“, die mit der Ankunft der ersten Kolonialbeamten und Missionare angebrochen war. Aus diesem Grund empfanden manche meiner Gewährsmänner und -frauen, viele davon praktizierende Christen, mein unermüdliches Interesse an der vorchristlichen Zeit als Paradox. Was kümmerten mich die alten, „heidnischen“ Vorstellungen und Praktiken, wo sie 
selbst das alles doch glücklich hinter sich gelassen hatten? Dieses Unverständnis sehe ich heute auch als eine verhüllte Kritik an einem zu stark rückwärts gewandten Forschungsfokus, der zu wenig auf aktuelle Verhältnisse und Probleme Bezug nahm. ${ }^{2}$

Ich sehe allerdings keine Anzeichen dafür, dass die Wahrnehmung eines paradoxen Interesses oder mein Priesterstatus meine Forschungen wesentlich behindert hätten. Dafür sehe ich aber heute sehr deutlich, dass meine missionarische Ausbildung und meine starke innere Verpflichtung auf die katholischen Prinzipien des Dialogs und der Inkulturation einen unaufdringlichen, aber nachhaltigen Einfluss auf meine Forschung sowie die Interpretation und Präsentation der Ergebnisse ausgeübt haben: Meine Analyse des vielschichtigen Christianisierungsprozesses betont - bei aller Differenzierung - Kontinuität mehr als Diskontinuität. Diese subjektiven Faktoren wirkten demnach nicht nur auf die Art der Wissensproduktion ein (Hauser-Schäublin 2002:92), sondern auch auf die Gewichtung und Darstellung ihrer Ergebnisse. Ich stimme meiner Erstgutachterin Hauser-Schäublin darin bei, dass eine andere Perspektive - und eine andere Sehgewohnheit - mit den gleichen Daten ein Bild mit mehr Brüchen und strukturellem Wandel ergeben hätte.

\section{Teilnahme, Beobachtung, Einmischung}

"Teilnehmende Beobachtung" trifft als spannungsgeladene Kurzformel nicht nur den Kern ethnologischer Feldforschung, sondern auch ihren inhärenten Widerspruch zwischen Nähe und Distanz - und dem "gefühlsmäßige[n] Oszillieren zwischen Beidem“ (Hauser-Schäublin 2003:38). Das damit gegebene ethische Problem der Einmischung ins „ungestörte ethnographische Leben“ thematisiert Hauser-Schäublin schon ganz früh (1976:13), weil ihr bereits ihre erste, sechsmonatige Feldforschung bei den Iatmul am Mittelsepik klarmacht, dass jeder Forschungsaufenthalt eine Einmischung ist und Veränderungen oder wenigstens die Sehnsucht danach bewirkt. Die implizite Frage lautet: Wie viel Einmischung ist legitim? Man kann m.E. auch andersherum fragen: Wie wenig Einmischung ist legitim, ethisch verantwortbar?

Durch meine doppelte Rolle als Ethnologe und Missionar stieß ich sehr bald an die engen Grenzen einer strikt teilnehmenden Beobachtung: bei christlichen Ritualhandlungen schon deshalb, weil ich solche oft initiierte und leitete, statt sie einfach teilnehmend zu beobachten. Das erforderte fast zwangsläufig die Reflexion - sowohl im Feld als auch danach im Schreibprozess - über die Angemessenheit einer Einmischung, meiner aktiven Mitwirkung an Änderungen. Die entscheidende Motivation und Legitimation für diese aktive Rolle lieferte mein Verständnis von Mission als respektvolle dialogische Begegnung zwischen der biblisch-christlichen Tradition mit ihrer ,Wahrheit' und ihren Werten und einer anderen kulturellen Tradition mit ihrer, Wahrheit' und ihren Werten. Für einen echten Dialog ist die grundsätzliche Achtung vor der anderen Tradition unerlässlich, und diese beinhaltet das aufmerksame Hinhören 
ebenso wie die Bereitschaft, von ihr zu lernen. Ich will nicht sagen, dass mir das immer gelungen ist, aber:

I was interested in fostering this dialogue and a respectful attitude towards the indigenous cultural tradition [...]. I thus encouraged Somaip Catholics to express their faith as much as possible in their own cultural ways and to reconsider their rhetoric that turned their pagan forebears into worshippers of Seten [Tok Pisin for "Satan"]. I found myself exploiting many possibilities (such as conversations, homilies, liturgical texts) to vindicate the ancestors, their knowledge and practices and thus to counter the pervasive trope of a dark, pagan past having given way to an enlightened Christian present. (Reithofer 2006:15)

Zwei Beispiele mögen meine missionarischen Interventionen, die ich durchaus als Beiträge oder Vorschläge zur Indigenisierung des Christentums verstand, veranschaulichen:

In Vorbereitung auf das Jubiläumsjahr 2000 regte ich in einem Workshop über Buße und Versöhnung katholische Gemeindemitglieder dazu an, neue und kulturell angemessenere Formen und Symbole für gemeinschaftliche Feiern der Versöhnung und Erneuerung zu entwickeln. Damit sollten auch Alternativen entstehen zu der in der katholischen Kirche vorherrschenden Form der Einzelbeichte, die m. E. einer kulturell wie theologisch unangemessenen Privatisierung des Glaubens Vorschub leistet. Es war dieser Workshop, der eine ungeahnte millenniale Dynamik entwickelte und am Vorabend der Jahrtausendwende zu einer Welle von Erneuerungsritualen führte, in denen mit großer Ernsthaftigkeit auch langlebige Konflikte in und zwischen den einzelnen Gemeinschaften in versöhnender Haltung thematisiert wurden (vgl. Reithofer 2006:324-328).

In anderen Fällen unterstützte ich indigene Initiativen, so etwa die Erstellung eines apologetischen Büchleins mit dem Titel „Tok i tru“ (Tok Pisin für „Wahrheit“), das katholischen Christen Argumentationshilfen anbot für interreligiöse Streitgespräche vor allem mit Adventisten, die diesbezüglich weit besser gerüstet waren. Es kostete mich einige innere Überwindung, Bibelstellen und andere Argumente zusammenzustellen gegen die üblichen adventistischen Anschuldigungen (Halten des Sonntags statt des Sabbats, fehlende Meidung von Alkohol, Tabak und Schweinefleisch, ,Verehrung' des Papstes und der Jungfrau Maria usw.). Was mich zur Mithilfe bewegte, war ein kultureller Lernprozess, die Erkenntnis nämlich, wie stark anschuldigende Worte wirken und empfunden werden (auch in anderen Bereichen des sozialen Lebens) und wie wichtig es für die Selbstachtung ist, hier Paroli bieten zu können.

Mitunter wirkte ich, bewusst und unbewusst, auch als Sand im Getriebe der Indigenisierung. So etwa wurde eine Pfingstpredigt, in der ich das Zungenreden zugunsten anderer Gaben des Heiligen Geistes (z.B. Weisheit, Friede) relativierte, als Angriff gegen eine bestimmte Form charismatischer Gebetsgottesdienste gedeutet, in denen Zungenreden eine prominente Rolle spielte (Reithofer 2006:299). Die Folge war, dass 
diese Laiengottesdienste eine längere Zeit ausgesetzt wurden, ohne dass ich den Grund dafür auch nur ahnte.

Die Beispiele meiner aktiven Einflussnahme werfen viele Fragen auf, von denen hier vor allem methodische und ethische relevant sind. Die heikelste Frage in methodischer Hinsicht hat in charakteristisch provokanter Manier Hauser-Schäublin auf den Punkt gebracht: "To what extent did I myself trigger what I describe as the Somaip indigenization of Christianity?” (Reithofer 2006:15).

Darauf kann ich Folgendes antworten: Die Indigenisierung ist zu einem überwiegenden Teil das Ergebnis vielfacher, auch widersprüchlicher indigener Interpretationen und langjähriger Aneignungsprozesse; ich habe aber in meiner Analyse versucht, meine Rolle und meinen Einfluss dort zu nennen, wo dies angebracht schien. Dabei sah ich jedoch eher die Gefahr der Überschätzung meines Einflusses. Ich machte meine pastorale Tätigkeit allerdings nicht zum expliziten Gegenstand meiner Untersuchungen. Heute denke ich, dass das doch in begrenztem Maße möglich gewesen wäre (etwa über indigene Mitarbeiter) und interessante Einblicke gewährt hätte.

Die gewichtigere Frage ist aber die nach der ethischen Rechtfertigung einer solchen Einmischung in lokale Angelegenheiten. Selbstverständlich wird eine Antwort darauf davon abhängen, ob man sich "nur" auf Ethnologen bezieht oder auf solche, die zugleich auch Missionare sind. Schließlich sind Aufgaben und Selbstverständnis eines Ethnologen nicht deckungsgleich mit denen eines Missionars. Der Steyler Missionar Philip Gibbs etwa, der auf eine jahrzehntelange Tätigkeit als missionary-researcher im Hochland von PNG zurückblickt (Gibbs 2004), sieht hinter der Ethnologen und Missionaren gemeinsamen Bereitschaft, mit Menschen anderer Kulturen das Leben zu teilen, unterschiedliche Motivationen am Werk: Bei Ethnologen die einer akademischen Qualifikation, bei Missionaren die des Bestrebens, zu den Menschen selbst, ihren Gedanken und Herzen zu gelangen und mit ihnen eine Glaubensbotschaft in einer sinnvollen Weise zu teilen (2004:61). Während ich mich dem zweiten Statement voll anschließen kann, halte ich das erste für pauschal und zu kurz gegriffen: Ich kenne viele Ethnologinnen und Ethnologen, deren Motivation sehr viel tiefgründiger und persönlicher ist als der Wunsch nach Titel oder Karriere. Des Weiteren sind die (in aller Regel) temporären, eher kurzfristigen Aufenthalte der Ethnologen gegenüber den eher langfristigen, oft auf unbestimmte Dauer angesetzten Aufenthalten der Missionare abzuwägen. Wo Missionare über Jahrzehnte ihr Leben mit Menschen einer anderen Kultur teilen, geht „Einmischung" ganz natürlich über in das, was man normale soziale Interaktion, Lebensgestaltung und politisches Engagement nennen kann.

Ungeachtet der Unterschiede glaube ich aber, dass es bestimmte ethische Kriterien gibt, die für beide Personengruppen relevant sind. Ich sehe Einmischung - insoweit sie nicht ohnehin durch bloße Anwesenheit schon gegeben ist - nicht per se als ethisch bedenklich an; auch nicht für Ethnologen. Wenn Feldforschung zwangsläufig verkörperte Feldforschung ist und die Forschungssituation ein kommunikatives und dialogisches Unterfangen zwischen so weit wie möglich gleichberechtigten Partnern, dann 
halte ich Intervention in bestimmten Situationen und Beziehungen für einen berechtigten, gar notwendigen Aspekt dieses Unterfangens, solange und sofern diese Ausdruck eines integren und aufrichtigen Umgangs miteinander ist, der die Würde und die Rechte des anderen respektiert. Um so mehr in jenen Fällen, in denen es sich um Langzeitforschungen und langfristig eingegangene Beziehungen und Verpflichtungen seitens der Forscher handelt.

Die ethisch entscheidende Frage (in Bezug auf Missionare als "change agents“) stellt m. E. Michael Rynkiewich:

The key question is not whether options are good or bad, not whether change should or should not take place, but rather what kinds of power and influence missionaries have, now and in the past, over the decision-making processes (1980:174).

Diese Machtfrage ist für Missionare wie für Ethnologen gleichermaßen relevant. Meiner Erfahrung nach setzen sich Missionare damit aber weniger auseinander als Ethnologen. ${ }^{3}$ Im folgenden Abschnitt gehe ich unter dem Gesichtspunkt der vielschichtigen Beziehungen im Feld noch näher auf diese Machtfrage ein.

\section{Feldforschung als „Gewebe von Beziehungen“}

Hauser-Schäublin bezeichnet Feldforschung als ein "Gewebe von Beziehungen“ (2002:73), in das Forscher temporär eingeflochten werden. In ihren einschlägigen Beiträgen macht sie sehr schön klar, dass das immer auch ein engmaschiges Gewebe von Machtverhältnissen, unterschiedlichen Erwartungen, Ansprüchen und Forderungen ist, also viel Konfliktpotenzial birgt. Sie betont dabei nicht nur, dass die Machtposition keineswegs eindeutig und einseitig beim Ethnologen liegt (2002:84f.), sondern auch den radikalen Umbruch, der sich in wenigen Jahrzehnten hinsichtlich der sozialen Beziehungen im Feld vollzogen hat: An die Stelle eines quasi-kolonialen Distanzverhältnisses, das dem Ethnologen einen Sonderstatus eingeräumt hat, ist eine Situation getreten, die beiden Seiten viele Spielräume offen lässt für die jeweilige Aushandlung gewünschter bzw. geforderter Nähe und Distanz, bis hin zum absoluten Distanzverlust (2002:78f.).

Ich selbst war froh über diese Spielräume, die es trotz meines Sonderstatus als "Stationspfarrer" erlaubten, ganz unterschiedliche Vertrauensbeziehungen aufzubauen und in unterschiedliche Familien eingebunden zu werden als Sohn, Bruder, für einige wenige auch als eine Art Vater. Auch Freundschaften entwickelten sich, die stark genug waren, bei allem, was uns einte und verband, auch das bleibende Trennende auszuhalten, die unterschiedliche Einstellung oder Bereitschaft zur Gewalt beispielsweise (ein Aspekt, mit dem auch Hauser-Schäublin gerungen hat). Meine Gastgeber - und hier an erster Stelle Frauen und Mütter - haben diese starke affektive Nähe und Gemeinsamkeit bei gleichzeitiger Anerkennung von Differenz in bewegender Weise ausgedrückt, 
als die Zeit meines Abschieds heranrückte. Die Form, die sie wählten, waren liwaip, kurze Textkompositionen zu einer charakteristischen Melodie, die mehrmals wiederholt wird. Das folgende Beispiel texteten Agnes Hoker und Jona Wal:
Kusipa kusi // Kusipa hanwa
Ich bin vom Kusipa-Fluss.
Father John o // 2000 mual ipis lo
Father John, dass du im Jahr 2000 kommen würdest,
nen nasape.
um zu helfen, wer hätte das gedacht?
Su gon e gon wuwa, heneng,
Ich habe [meine Gedanken] auf diese Welt, auf dieses Feld,
and gon wuwa, amen.
auf dieses Haus gerichtet. Amen.
Solwara moisal // hondapenja la.
Geh geradewegs über das Meer!
Haki, yan bali nenga senal,
Schwester, ist es unser kleiner Bruder,
wal ip lopa pe, heneng.
dass wir sagen könnten: "Komm wieder!"?
Bali, gen hame nenga senal
Bruder, ist es dein kleiner Bruder,
wal peke losi, amen.
dass du sagen könntest: "Komm zurück!"?

Ich bin für Menschen dort wie ein Bruder geworden, aber doch nicht so, dass die Bande zwischen uns so belastbar gewesen wären wie zwischen wirklichen Geschwistern. Mein bevorstehender Abschied rückte diese Tatsache klar ins Bewusstsein. Weil auch für mich viele der Menschen wie Brüder und Schwestern, Väter und Mütter geworden waren, empfand ich diese Differenz zu wirklichen familiären Beziehungen ebenfalls sehr stark, und es war mir ein Bedürfnis, meine Rückkehr „in spätestens drei Jahren“ zu versprechen, um so mir und den anderen die Stärke unserer Verbindung zueinander (und meiner Gefühle für sie) gewissermaßen zu beweisen. Auch in der Absicht, ganz augenscheinliche Unterschiede zwischen uns zu relativieren, auf die sich etwa ein anderes Abschiedslied bezog: „Bruder, wenn wir die gleiche Hautfarbe hätten, würde ich sagen: Geh nicht, geh nicht!" Ich erkenne neidlos an, dass die Somaip mir weit überlegen waren in der Fähigkeit, die Trauer über den Abschied in Worte und Bilder zu fassen; ein gutes Beispiel dafür ist die folgende Komposition einer Frauengruppe:
Profet John o // peou peou lel
Prophet John, du sagst: "Ich gehe."
bur isi mupa lo // nen sapo haiyo.
Mein erstgeborener Sohn, meine
Gedanken sind bei dir.
And go ne go neou,
Wenn ich in diesem Haus hier esse,
tamp naepe o, heneng.
merke ich den Geschmack des Essens nicht. 
Ugopaileou,

unaepe, amen.
Wenn ich mich zum Schlafen hinlege,

kommt der Schlaf nicht. Amen.

Die Tatsache, dass Ethnologen kommen und (wieder) gehen, ganz egal, wie lange sie bleiben und wie eng sie ihre sozialen Beziehungen mit den Gastgebern knüpfen, ist zunächst etwas, mit dem beide Seiten fertig werden müssen. Es unterscheidet Ethnologen, wie schon angedeutet, von Missionaren. Diese Tatsache des begrenzten Aufenthalts muss dann aber auch in ethischer Verantwortung mit berücksichtigt werden in der Gestaltung der sozialen Beziehungen. Diese Tatsache reflektiert auch ungleiche Machtverhältnisse und Lebensgestaltungsmöglichkeiten: Die Länge des Aufenthalts und den Zeitpunkt der Abreise bestimmt in aller Regel der Forscher selbst (freilich in Abhängigkeit von Stipendiengebern und anderen übergeordneten Zeitzwängen) und nimmt dabei Rücksicht auf seine Forschungspläne und Erwartungen, und selten auf die seiner Gastgeber. Was wird aus ihren Erwartungen und Hoffnungen?

Ich habe mich das oft gefragt, weil mein fürstlicher Empfang in Ipisam, wie eingangs angemerkt, erkennen ließ, dass man große Hoffnungen in mich setzte. Bei meinem re-visit im Jahr 2003, als ich meine fast fertige Studie besprechen und meine (!) noch offenen Fragen klären wollte, wurde mir mitgeteilt, dass diese Hoffnungen in zwei Richtungen gegangen waren, die aber ganz fundamental zusammengehörten wie die zwei Seiten einer Medaille. Katechist Henry Palip brachte die Schieflage der erfüllten und enttäuschten Hoffnungen deutlich genug zum Ausdruck:

This book is about the "side of belief" of our ancestors and ourselves as Christians. But on the "side of the body" we lack almost everything: We have no school, no aid post, no road, no market [...]. (Palip zitiert in Reithofer 2006:339)

Mein aus der Forschung hervorgegangenes Buch hatte nur der ,Seite des Glaubens Rechnung getragen - dadurch etwa, dass es das reiche und komplexe rituelle und mythologische Leben der vorchristlichen Ära (so gut es ging) rekonstruiert und so auch bewahrt. Dabei muss ich eingestehen, dass diese westliche Art der Bewahrung einer Vergangenheit zwischen Buchdeckeln nur von wenigen Somaip gewürdigt und wertgeschätzt wurde und wird. ${ }^{4}$ Auf der ,Seite des Körpers' aber hatte sich praktisch nichts bewegt; die Region war nach wie vor in sozioökonomischer und politischer Hinsicht desolat aufgestellt, vernachlässigt. Gerade die Christen unter den Somaip empfanden darüber Unmut und Enttäuschung: Was hatten ihnen die Übernahme des Christentums und das Bemühen um ein friedliches, christliches Leben eingebracht? Für mich schwang in dieser Frage auch mit: Was hatte ihnen mein zweijähriger Aufenthalt in ,körperlicher' Hinsicht eingebracht? Hatten sie sich von mir auch diesbezüglich (mehr) Einmischung und Wirkung erhofft? Was sie mir gegenüber aber ausdrückten, war die Hoffnung, die sie an die Veröffentlichung meines Buches knüpften: 
They communicated to me their hope that this book could help to convince members of the (provincial) government that there are "good Christian" clans in Honda worthy of support and the allocation of development projects, so that the "body side" might once again correspond more closely with the "belief side". (Reithofer 2006:339)

Wohl jeder Ethnologe, der Feldforschung gemacht hat, kennt diese Erfahrung: dass er Hoffnungen weckt, die er nicht erfüllen kann, dass er Menschen enttäuscht, die ihm vertraut und mehr zugetraut haben, als er dann fähig oder willens war zu „liefern“. Diese Erfahrung wird sich kaum vermeiden lassen. Warum mir aber diese Diskussion mit meinen Gastgebern noch so lebendig in Erinnerung ist, und worauf es mir hier ankommt, sind die Indizien asymmetrischer Machtverhältnisse. Ich hatte mein Forschungsthema noch vor meiner Ankunft bestimmt und dann auch durchgezogen (und bin dann wieder abgezogen). Ich habe gegenüber meinen Gastgebern den ideellen Nutzen einer solchen Studie hervorgehoben, aber mich nicht gefragt, ob die Erforschung einer anderen Thematik aus indigener Perspektive dringender und auch nützlicher wäre. In dieser Hinsicht bin ich wohl vielen Ethnologen sehr viel ähnlicher als etwa ethnologisch forschenden Missionaren.

Missionary-researcher Philip Gibbs etwa hält es für unvertretbar, in der gegenwärtigen Situation in PNG apolitische Forschungen zu unternehmen unabhängig von den Interessen der verschiedenen Gruppen in der Gesellschaft (2004:76). Dafür gebe es einfach zu viele große und drängende Herausforderungen, die der gemeinsamen Anstrengung von Missionaren und Ethnologen bedürften, um wichtige Erkenntnisse zu gewinnen und effektive, kulturell adäquate Problemlösungen zu entwickeln (2004:78). Ganz ähnlich auch der Steyler Missionar Douglas Young, mittlerweile Erzbischof der Diözese Mt. Hagen (= Western Highlands Province), der in seinen Forschungen über gewaltfreie Konfliktlösungsstrategien in Enga einen expliziten participatory action research-Ansatz verfolgte (Young 2004) und generell ein Mitspracherecht der Gastgeber bei der Wahl der Forschungsthematik einfordert.

\section{Schluss}

Kommen wir also zur angekündigten häretischen Frage: Sind Missionare (manchmal) die besseren Ethnologen? Ich beginne defensiv. Wenn wir von einem Missionsverständnis ausgehen, bei dem die Achtung der jeweiligen kulturellen Tradition und Situation selbstverständlich und „Dialog“ das Schlüsselprinzip ist, dann kann ich hier eine grundsätzliche Kompatibilität, wenn nicht Gemeinsamkeit, feststellen mit dem ethnologischen Unterfangen der Feldforschung, in der sich - um mit Pat Caplan zu sprechen - eine radikale Wende von einer „observational and empirical methodology to a communicative and dialogical epistemology" (zitiert nach Hauser-Schäublin 2002:92) vollzogen hat. Ich sehe für diese dialogische Begegnung nicht den unbedingten Vorteil einer (angeblich) wertneutralen Position gegenüber einer Wertepos- 
ition, die ja keineswegs ein echtes Verstehen-Wollen und eine Achtung des Anderen ausschließt. ${ }^{5}$ Der echte interreligiöse Dialog (um ein nicht-ethnologisches Beispiel zu nennen) lebt ja gerade vom Ernstnehmen der eigenen Überzeugung und der aus dem Glauben bezogenen Werteposition und vom gleichzeitigen Ernstnehmen der Überzeugungen und Werte der jeweiligen Dialogpartner. Der schwedische lutherische Missionar Bengt Sundkler hält es für ehrlicher und wichtiger, die eigene bias, die ohnehin unausweichlich gegeben ist, zu kennen und zu benennen, statt so zu tun, als gäbe es eine völlig wertneutrale, unvoreingenommene Sozialwissenschaft (Bowie 2000:7-8). Ein ganz ähnliches Anliegen sehe ich in der oben schon erwähnten Forderung Hauser-Schäublins (1976:9) nach persönlichen Rechenschaftsberichten artikuliert. Methodisch gesehen ergeben sich für einen ethnologisch forschenden Missionar in aller Regel besondere Probleme und Herausforderungen, auch in Abhängigkeit des gewählten Forschungsbereichs, aber diese sind keineswegs unlösbar oder verurteilen von vornherein das ethnographische Unterfangen zum Scheitern.

Was die aktive Einflussnahme oder - weniger euphemistisch - die Einmischung in das Leben der Gastgebergesellschaft seitens des Forschers angeht und deren ethische Beurteilung, so habe ich oben argumentiert, beiden Personengruppen (in unterschiedlichem Maße und in Abhängigkeit verschiedener Faktoren) das Recht auf Einmischung zuzugestehen. Dabei bleibt die Machtfrage, wie sie Rynkiewich formuliert hat, kritisch und unentbehrlich.

An dieser Stelle bietet sich der Wechsel zu einer offensiven Beantwortung der Frage an. Hauser-Schäublin greift in ihrem Beitrag über „Teilnehmende Beobachtung “ Gerd Spittlers (2001) Ausführungen über „dichte Teilnahme“ auf, die als soziale Nähe immer auch Empathie, Einfühlen und Mitfühlen beinhalte und Voraussetzung für ein vertieftes Verstehen sei (Hauser-Schäublin 2003:38f.). Wenn dem zugestimmt werden kann, dann lassen sich m. E. schon vorteilhaftere Bedingungen für ethnologisch forschende Missionare ausmachen, sofern wir von einem langfristigen Zusammenleben und von dialogbereiten Menschen ausgehen. Gibbs bemerkt, nach vielen Jahren des Zusammenlebens mit Menschen in der Enga Province (PNG), eine fundamentale Änderung seiner Wahrnehmung und seines Blickwinkels: „This Enga society, so foreign and strange initially, was now ,home ““ (2004:69). Für mich ist Gibbs ein gutes Beispiel dafür, dass going native keine ethnologische Katastrophe bedeuten muss, sondern eher das Gegenteil heißen kann. Aus dieser intimen und große Zeiträume umspannenden Kenntnis der Menschen kann ein hohes Maß an Involviertsein, Engagement und Identifikation erwachsen - und die Forderung nach einer ethnologischen Forschung, die sich von den Interessen und Problematiken der betreffenden Gastgebergesellschaften leiten lässt. Wer diesen Grundgedanken der kooperativen Forschung für die Ethnologie insgesamt wenigstens für bedenkenswert hält, wird diese Einstellung auch bei Forscher-Missionaren zu würdigen wissen.

Können Missionare also die besseren Ethnologen sein? Ich werde jetzt doch den einen oder anderen Leser enttäuschen; denn ich möchte die Frage in dieser Form gar 
nicht beantworten, weil sie von einem Gegeneinander und einer Konkurrenz ausgeht, die ich gerade überwinden will. Es geht darum, die Stärken und Schwächen, Gefahren und Chancen - die eigenen und die der anderen - deutlicher zu sehen. Und es geht darum, die Komplementarität und die Notwendigkeit der Zusammenarbeit zu sehen, weil die drängenden Herausforderungen unserer Zeit einen multidisziplinären Ansatz brauchen, der missionarischen Eifer ebenso umfasst wie akademische Erkenntnissuche (Gibbs 2004:78). Es gibt gottseidank genug Ethnologen und genug ethnologische Ansätze, die sich gerade diesen drängenden Herausforderungen stellen wollen.

Für mich ist Hauser-Schäublin eine davon. Ihre Forschungen, die sie seit 1988 auf Bali durchführt, haben einen unverkennbar kooperativen Charakter (z. B. HauserSchäublin 2008), ihre Themen in Lehre und Forschung reflektieren ein hochpolitisches und waches Bewusstsein für die großen Herausforderungen unserer Zeit. Ich möchte nur ein Erlebnis nennen, in dem sie, wie mir scheint, einen Blick gewährt hat auf ihre zentrale Motivation für ihre ethnologischen Forschungen. Es war der Satz, in eine Vorstandssitzung über Lehrprogrammplanung hineingesprochen: „Für mich brennt die Welt!" Um die Brandherde zu löschen oder wenigstens einzudämmen, braucht es auch - und nicht zuletzt - Ethnologinnen und Ethnologen.

Als sich Hauser-Schäublin in einer Feldforschungssituation vor einigen Jahren für ein Gespräch auf den Boden setzen wollte, wurde sie von der Gastgeberin höflich, aber bestimmt daran gehindert mit den Worten: „Alte Leute müssen sich aufs Sofa setzen“ (Hauser-Schäublin 2002:74). Auch wenn in dieser Fürsorge viel Respekt mitschwang, möchte ich selbst diese Wahrnehmung des vorgerückten Alters doch zurückweisen und meiner Hoffnung Ausdruck verleihen, dass sie ihren sogenannten Ruhestand nicht zum Anlass nimmt, sich von nun an aufs Sofa zu setzen und auszuruhen.

\section{Anmerkungen}

1 Umso dankbarer bin ich für die sehr fruchtbare und vertrauensvolle Zusammenarbeit mit einigen ausgewählten und ihrer Kirche gegenüber sehr loyalen Adventisten.

2 Ich sehe hier eine gewisse Parallele mit dem Konflikt, den Hauser-Schäublin durch ihre Forschungen unter den Abelam hervorrief: Ihr Interesse am traditionellen Wissen erregte den Widerstand von Mitgliedern der jüngeren Generation, deren Trachten mehr auf Fortschritt und Geld gerichtet war (Hauser-Schäublin 1985:186f.).

3 Das gilt nur bedingt für die Steyler Missionare, bei denen ich generell ein sehr waches Bewusstsein für diese Frage erlebte.

4 Weniger jedenfalls, als dies für Hauser-Schäublin und ihre Forschungen auf Bali zuzutreffen scheint. In Traces of Gods and Men schreibt sie: „But what really drove me to my desk were all those Balinese who told me what they know about [...]. They entrusted their knowledge to me with request to publish and thereby preserve it." (1997:9)

5 Mit Freude las ich im Erstgutachten von Hauser-Schäublin über meine Dissertation den Satz: „Aus seinen Beschreibungen der vorchristlichen Religion der Somaip spricht eine tiefe Bewunderung und Faszination $[\ldots]$... 


\section{Literatur}

Bowie, Fiona (2000) The Anthropology of Religion. An Introduction. Oxford: Blackwell Publishers.

Fischer, Hans (2002) Feldforschungen. Erfahrungsberichte zur Einführung (2. Auflage, Neufassung). Berlin: Reimer.

Gibbs, Philip (2004) Changing Perceptions of a Missionary-Researcher. Pacific Studies $27(3 / 4): 61-80$.

Hauser-Schäublin, Brigitta (1976) Feldforschung bei den Iatmul: Zwischen Erlebnis und Wissenschaft. Bulletin der Schweizerischen Ethnologischen Gesellschaft, Sondernummer „Probleme der Feldforschung aus der Sicht junger Ethnologen“:9-14.

Hauser-Schäublin, Brigitta (1977) Frauen in Kararau. Zur Rolle der Frau bei den Iatmul am Mittelsepik, Papua New Guinea. Basel: Ethnologisches Seminar der Universität und Museum für Völkerkunde (Basler Beiträge zur Ethnologie 18).

Hauser-Schäublin, Brigitta (1985) Frau mit Frauen. Untersuchungen bei den Iatmul und Abelam, Papua Neuguinea. In: Hans Fischer (Hg.): Feldforschungen. Berichte zur Einfuibrung in Probleme und Methoden, S. 179-201. Berlin: Reimer.

Hauser-Schäublin, Brigitta (1997) Traces of Gods and Men. Temples and Rituals as Landmarks of Social Events and Processes in a South Bali Village. Berlin: Reimer.

Hauser-Schäublin, Brigitta (2002) Gender: Verkörperte Feldforschung. In: Hans Fischer (Hg.): Feldforschungen: Erfahrungsberichte zur Einführung (2. Auflage, Neufassung), S.73-100. Berlin: Reimer.

Hauser-Schäublin, Brigitta (2003) Teilnehmende Beobachtung. In: Bettina Beer (Hg.): Methoden und Techniken der Feldforschung, S.33-54. Berlin: Reimer.

Hauser-Schäublin, Brigitta und I Wayan Ardika (2008) Burials, Texts and Rituals. Ethnoarchaeological Investigations in North Bali, Indonesia. Göttingen: Universitätsverlag (Göttinger Beiträge zur Ethnologie 1).

Kocher Schmid, Christin (1999) Expecting the Day of Wrath. Versions of the Millennium in Papua New Guinea. Boroko: The National Research Institute (NRI Monograph 36).

Reithofer, Hans (2006) The Python Spirit and the Cross. Becoming Christian in a Highland Community of Papua New Guinea. Berlin: Lit (Göttinger Studien zur Ethnologie 16).

Rynkiewich, Michael (1980) Response to „Anthropologists versus Missionaries: The Influence of Presuppositions", by Claude E. Stipe. Current Anthropology 21(2):165179. 
Spittler, Gerd (2001) Teilnehmende Beobachtung als Dichte Teilnahme. Zeitschrift für Ethnologie 126(1):1-25.

Young, Douglas W. (2004) „Our Land is Green and Black“. Conflict Resolution in Enga. Goroka, Papua New Guinea: Melanesian Institute (Point Series 28). 